\title{
Aortic stiffness in aortic stenosis assessed by cardiovascular MRI: a comparison between bicuspid and tricuspid valves
}

\author{
Anvesha Singh ${ }^{1}$ (D) Mark A. Horsfield ${ }^{2}$. Soliana Bekele ${ }^{1}$. John P. Greenwood ${ }^{3}$. Dana K. Dawson ${ }^{4} \cdot$ Colin Berry $^{5}$. \\ Kai Hogrefe ${ }^{6}$. Damian J. Kelly ${ }^{7}$. John G. Houston ${ }^{8} \cdot$ Prasad Guntur Ramkumar $^{8} \cdot$ Akhlaque Uddin $^{3} \cdot$ Toru Suzuki $^{1}$. \\ Gerry P. McCann ${ }^{1}$
}

Received: 14 May 2018 / Revised: 3 August 2018 / Accepted: 19 September 2018 / Published online: 28 November 2018

(C) The Author(s) 2018

\begin{abstract}
Objectives To compare aortic size and stiffness parameters on MRI between bicuspid aortic valve (BAV) and tricuspid aortic valve (TAV) patients with aortic stenosis (AS).

Methods MRI was performed in 174 patients with asymptomatic moderate-severe AS (mean AVAI $0.57 \pm 0.14 \mathrm{~cm}^{2} / \mathrm{m}^{2}$ ) and 23 controls on 3T scanners. Valve morphology was available/analysable in 169 patients: 63 BAV (41 type-I, 22 type-II) and 106 TAV. Aortic cross-sectional areas were measured at the level of the pulmonary artery bifurcation. The ascending and descending aorta (AA, DA) distensibility, and pulse wave velocity (PWV) around the aortic arch were calculated.

Results The AA and DA areas were lower in the controls, with no difference in DA distensibility or PWV, but slightly lower AA distensibility than in the patient group. With increasing age, there was a decrease in distensibility and an increase in PWV. After correcting for age, the AA maximum cross-sectional area was higher in bicuspid vs. tricuspid patients $(12.97[11.10,15.59]$ vs. $\left.10.06[8.57,12.04] \mathrm{cm}^{2}, p<0.001\right)$, but there were no significant differences in AA distensibility $(p=0.099)$, DA distensibility $(p=0.498)$ or PWV $(p=0.235)$. Patients with BAV type-II valves demonstrated a significantly higher AA distensibility and lower PWV compared to type-I, despite a trend towards higher AA area.

Conclusions In patients with significant AS, BAV patients do not have increased aortic stiffness compared to those with TAV despite increased ascending aortic dimensions. Those with type-II BAV have less aortic stiffness despite greater dimensions. These results demonstrate a dissociation between aortic dilatation and stiffness and suggest that altered flow patterns may play a role.

Key Points

- Both cellular abnormalities secondary to genetic differences and abnormal flow patterns have been implicated in the pathophysiology of aortic dilatation and increased vascular complications associated with bicuspid aortic valves (BAV).

- We demonstrate an increased ascending aortic size in patients with BAV and moderate to severe AS compared to TAV and controls, but no difference in aortic stiffness parameters, therefore suggesting a dissociation between dilatation and stiffness.

- Sub-group analysis showed greater aortic size but lower stiffness parameters in those with BAV type-II AS compared to BAV type-I.
\end{abstract}

Electronic supplementary material The online version of this article (https://doi.org/10.1007/s00330-018-5775-6) contains supplementary material, which is available to authorized users.

Anvesha Singh

as707@le.ac.uk

1 Department of Cardiovascular Sciences, University of Leicester and Cardiovascular Theme, NIHR Leicester Biomedical Research Centre, Glenfield Hospital, Groby road, Leicester LE3 9QP, UK

2 Xinapse Systems Ltd., West Bergholt, Essex CO6 3BW, UK

3 Multidisciplinary Cardiovascular Research Centre \& The Division of Biomedical Imaging, Leeds Institute of Cardiovascular \& Metabolic Medicine, Leeds University, Leeds LS2 9JT, UK
4 Cardiovascular Medicine Research Unit, School of Medicine and Dentistry, University of Aberdeen, Polwarth Building, Foresterhill, Aberdeen AB25 2ZD, UK

5 BHF Glasgow Cardiovascular Research Centre, University of Glasgow, 126 University Place, Glasgow G12 8TA, UK

6 Cardiology Department, Kettering General Hospital Foundation Trust, Rothwell Rd, Kettering NN16 8UZ, UK

7 Cardiology Department, Royal Derby Hospital, Uttoxeter Rd, Derby DE22 3NE, UK

8 Division of Molecular \& Clinical Medicine, Ninewells Hospital and Medical School, Dundee DD1 9SY, UK 
Keywords Aortic valve stenosis $\cdot$ Pulse wave velocity $\cdot$ Aorta, thoracic $\cdot$ Aortic valve, bicuspid $\cdot$ Magnetic resonance imaging

\author{
Abbreviations \\ AA Ascending aorta \\ AS Aortic stenosis \\ BAV Bicuspid aortic valve \\ DA Descending aorta \\ LGE Late gadolinium enhancement \\ LV Left ventricular \\ MRI Magnetic resonance imaging \\ PP Pulse pressure \\ PWV Pulse wave velocity \\ TAV Tricuspid aortic valve
}

\section{Introduction}

Bicuspid aortic valve (BAV) is the most common congenital cardiac anomaly, affecting 1-2\% of the general population [1]. It is associated with an increased incidence of aortic root dilatation [2] and vascular complications, with the reported pooled risk of aortic dissection being as high as $4 \%[3,4]$. Cellular abnormalities, such as cystic medial necrosis and apoptosis, have been observed in the aortic walls of patients with BAV disease [5, 6]. Two dominant hypotheses have been proposed to explain these abnormalities: (i) the changes are due to a primary aortopathy which is genetically mediated $[5,6]$ and (ii) abnormal flow patterns associated with BAV disease lead to secondary changes in the aorta $[7,8]$.

The viscoelastic properties of the aorta are essential to maintain proximal and distal arterial flow and organ perfusion, and arterial stiffness has been shown to be independently associated with the development of cardiovascular disease [9]. Pulse wave velocity (PWV), which is the rate of propagation of the systolic wave front down a vessel, is a marker of vessel wall stiffness and inversely related to its distensibility. It can most accurately be measured invasively by intra-arterial pressure measurements; however, its non-invasive assessment is more practical. Magnetic resonance imaging (MRI) allows direct (aortic distensibility) and indirect (PWV) measurement of arterial stiffness in a single examination, at multiple sites, with good agreement compared to invasive measurements and with excellent reproducibility [10]. Abnormalities of aortic stiffness have been found in patients with normally functioning BAVs. Lower aortic distensibility and higher aortic stiffness index on echocardiography or PWV on MRI have been demonstrated in patients with BAV compared to controls [11-14]. The interpretation of these findings was that there is an intrinsic aortopathy associated with BAV.
However, no studies to date have reported on whether there are significant differences in aortic stiffness parameters in patients with aortic stenosis (AS) secondary to BAV disease and degenerative AS of a tricuspid valve (TAV). If patients with BAV did have a genetic predisposition to the development of aortopathy, one would expect stiffness parameters to be increased in comparison to those with TAV. We hypothesised that patients with AS and BAV would have significantly greater thoracic aortic stiffness measured by MRI than patients with tricuspid AS and healthy controls without valve disease.

\section{Materials and methods}

\section{Study population}

Asymptomatic patients with moderate to severe AS were prospectively recruited as part of a multicentre study conducted in the UK (PRIMID-AS study) [15]. Patients with more than mild degree of other valve disease, including aortic regurgitation, were excluded. Asymptomatic controls with no valve disease were also recruited for comparison.

\section{Ethics, consent, and permissions}

The study was approved by the United Kingdom National Research Ethics Service (11/EM/0410) and written informed consent was obtained from all subjects before participation.

\section{Echocardiography}

All subjects underwent a comprehensive trans-thoracic echocardiogram as per international guidelines, to quantify AS severity. In addition, mitral inflow velocities and tissue Doppler imaging (TDI) was used to assess diastolic function. Pulsed-wave Doppler was performed at the mitral valve tips to calculate the $\mathrm{E} / \mathrm{A}$ ratio, and pulsed-wave TDI was used to measure the septal and lateral mitral annular velocity, for calculation of the septal and lateral E/e'.

\section{MRI image acquisition}

Cine imaging was performed as previously described [16] on $3 \mathrm{~T}$ scanners to determine left ventricular (LV) volumes, mass and function. In addition, steady-state free precession (SSFP) cine images of the aortic valve (or gradient-echo if significant artefacts were present), and a high temporal resolution cine image of the 
ascending (AA) and descending aorta (DA) at the level of the pulmonary artery bifurcation (slice thickness $6 \mathrm{~mm}$, reconstructed to 40 phases, temporal resolution $\sim 20 \mathrm{~ms}$, matrix $192 \times 256$ ), were acquired. These cine images were used to measure aortic cross-sectional areas throughout the cardiac cycle and calculate distensibility [17] (Fig. 1). A retrospectively gated phase-contrast velocity-encoded sequence (typical parameters: slice thickness $5 \mathrm{~mm}$, VENC $250 \mathrm{~cm} / \mathrm{s}$, reconstructed to $100-128$ phases, temporal resolution $\sim 10 \mathrm{~ms}$, TE $4 \mathrm{~ms}$, matrix $176 \times 256$ ), optimised for the study with high temporal resolution and a large number of reconstructed phases, was acquired perpendicular to the ascending and descending thoracic aorta to calculate through-plane flow. As the planning of the slice was far away from the aortic valve, a VENC of $250 \mathrm{~cm} / \mathrm{s}$ was adequate in most cases, but if aliasing artefact was noted, then a repeat acquisition with a slightly higher VENC was performed. Brachial artery blood pressure was recorded at the time of the aortic cine acquisition to calculate the pulse pressure. A sagittal oblique view of the aortic arch was acquired to calculate the distance between sections for flow measurements in the ascending and descending aorta (Fig. 2). In addition late gadolinium enhancement (LGE) and pre- and post-contrast T1 mapping was performed for calculation of extracellular volume fraction, as previously described [16, 18].

\section{MRI image analysis}

All analyses were performed offline at the core lab, blinded to patient details, by a single observer (AS). LV volume and function were assessed using cvi42 V5 (circle cardiovascular imaging), excluding papillary muscle and trabeculations from the myocardial mass [19]. Valve morphology was classified according to Schaefer [20] with fusion of left and right coronary cusps in type-I BAV and right and non-coronary cusps in type-II BAV patients (there were no type-III patients in this cohort). Aortic root dimensions were measured at the annulus, sinus of Valsalva, sinotubular junction and proximal AA on the standard 3-chamber and the coronal LVOT views and an average was taken. Ascending and descending maximum and minimum aortic areas were measured from the aortic cine at the pulmonary artery bifurcation level. Aortic distensibility (in $10^{-3} \mathrm{mmHg}^{-1}$ ) was calculated using the following equation $[14,17]$ :

Distensibility $=[(A \max -A \min ) /(A \min \times \mathrm{PP})]$,

where Amax and Amin are the maximum and minimum aortic cross-sectional areas, and PP is the pulse pressure, i.e., systolic blood pressure - diastolic blood pressure $(\mathrm{mmHg})$.

PWV was assessed in the segment including the ascending aorta, the aortic arch and the proximal descending aorta up to the level of the pulmonary artery bifurcation (Fig. 2). PWV (in $\mathrm{m} / \mathrm{s}$ ) was calculated from:

$\mathrm{PWV}=\Delta x / \Delta t$,

where $\Delta x$ is the distance around the aortic arch between the two sections through the ascending and descending aorta, and $\Delta t$ is the transit time delay of two volume flow rate curves for the descending and ascending aorta. The sagittal oblique view of the aorta was used to measure the distance around the aortic $\operatorname{arch}(\Delta x)$, taking the mean of the two distance measures for the outer and inner borders of the aortic lumen. The software package 'Jim' (Version 7, Xinapse Systems) was used to calculate $\Delta t$ from the volume flow rate curves from the $\mathrm{AA}$ and DA. A maximum in the cross-correlation between these curves was used to estimate the transit time of the pressure wave around the arch.

\section{Statistical analysis}

Statistical tests were performed using SPSS 24.0 software (Statistical Package for the Social Sciences). Normality was assessed using the Shapiro-Wilk test, histograms and Q-Q
Fig. 1 End-diastolic (a) and endsystolic (b) frames from SSFP cine image of the ascending (top larger region) and descending (bottom smaller region) aorta, used for measurement of aortic dimensions and distensibility calculation
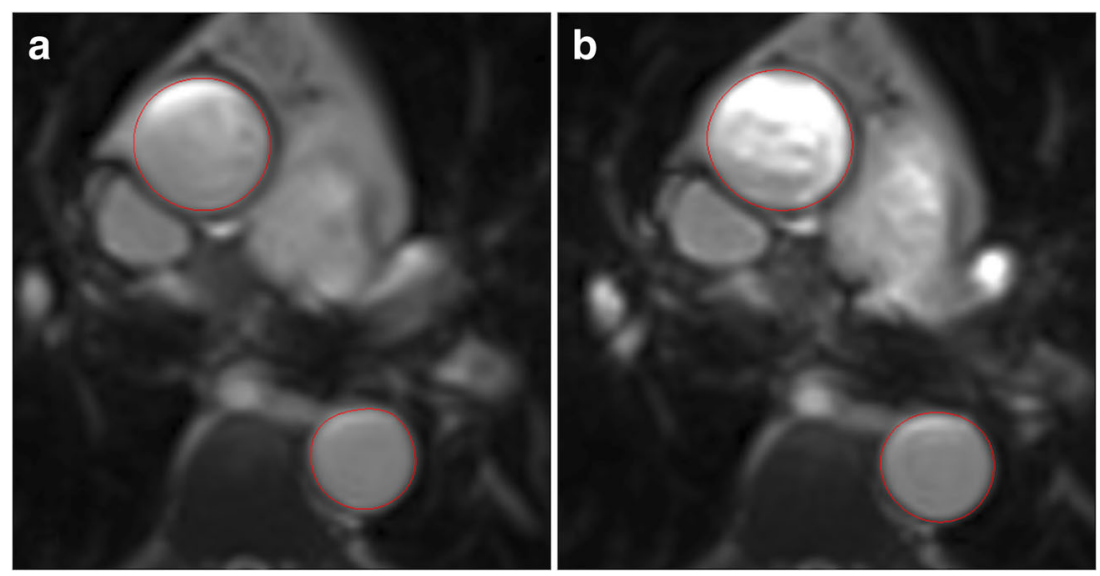
plots. For continuous data, summary statistics are expressed as mean \pm standard deviation. Log transformation was performed for non-normally distributed data. An independent sample $t$ test was used to compare normally distributed or log-transformed data between bicuspid and tricuspid patients, and between patients and controls. The Mann-Whitney test was used for non-parametric data comparison. The bicuspid group was further split by their sub-types (type-I, and type-II) and compared to tricuspid patients, using ANCOVA modelling, adjusted to take age into account. All $p$ values $<0.05$ were considered statistically significant. Univariate associations of distensibility and PWV were explored using linear regression analysis. Variables were then selected to be entered into a stepwise multivariable model based on statistical significance $(p<0.05)$ or clinical importance, whilst avoiding colinear variables.

\section{Results}

\section{Comparison of AS with controls}

Of 174 patients recruited, aortic valve morphology classification was possible in 169 (due to missing or unanalysable aortic valve cine imaging in 5 patients), with $63 \mathrm{BAV}$ and $106 \mathrm{TAV}$ patients forming the final population. Distensibility was not measurable in six of these patients due to aortic cine images not being available, and PWV was missing in eight due to missing flow images or artefacts. There was no difference in age and gender distribution between the 23 controls and overall AS group (Table 1). Patients had a higher incidence of hypertension and hyperlipidaemia, although treated hypertension was not excluded from the control group, to assess the incremental effect of AS on LV remodelling. AS patients demonstrated significantly higher LV volumes, mass and mass/volume and more LGE than controls. The aortic root measurements were higher in patients than controls at the annulus and proximal AA levels. The AA and DA areas were higher in patients, with no difference in DA distensibility or PWV, but slightly higher AA distensibility (Table 2).

\section{Comparison between bicuspid and tricuspid groups}

\section{Baseline characteristics}

The BAV group was significantly younger, with a lower incidence of hypertension and statin use and higher diastolic blood pressure than those with tricuspid AS (Table 1). The TAV group also had higher mean pressure gradient but similar peak gradient and aortic valve area index. Both groups had similar degree of LV remodelling, late gadolinium enhancement and extracellular volume fraction. The aortic root diameters were larger in BAV than TAV group at all levels (Table 1).

\section{Aortic area, distensibility and PWV}

The maximum and minimum cross-sectional AA areas were significantly higher in bicuspid patients and this remained statistically so even after correcting for age (Table 2). There was no difference in the age-corrected DA cross-sectional area. With increasing age, there was a decrease in distensibility ( $r=-0.45, p<0.001$ for the AA; $r=-0.64, p<0.001$ for DA) and an increase in PWV $(r=0.38, p<0.001)$ (Fig. 3ac). After correcting for age, there was no significant difference a

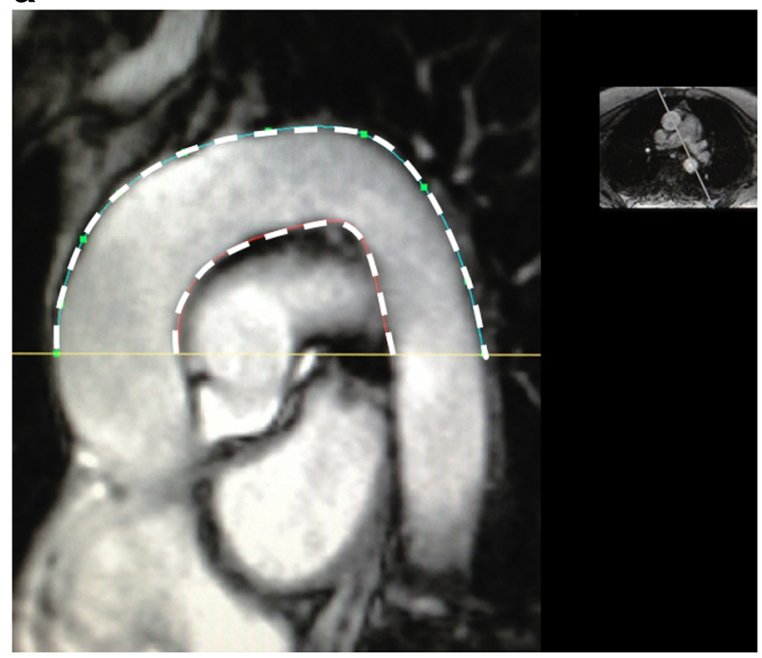

Fig. 2 Pulse wave velocity calculation. a Sagittal oblique cine of the aorta for measurement of $\Delta x$ (average of outer and inner distance in white dashed line). $\mathbf{b}$ Aortic flow sequence used for calculation of $\Delta t$, i.e., the

b

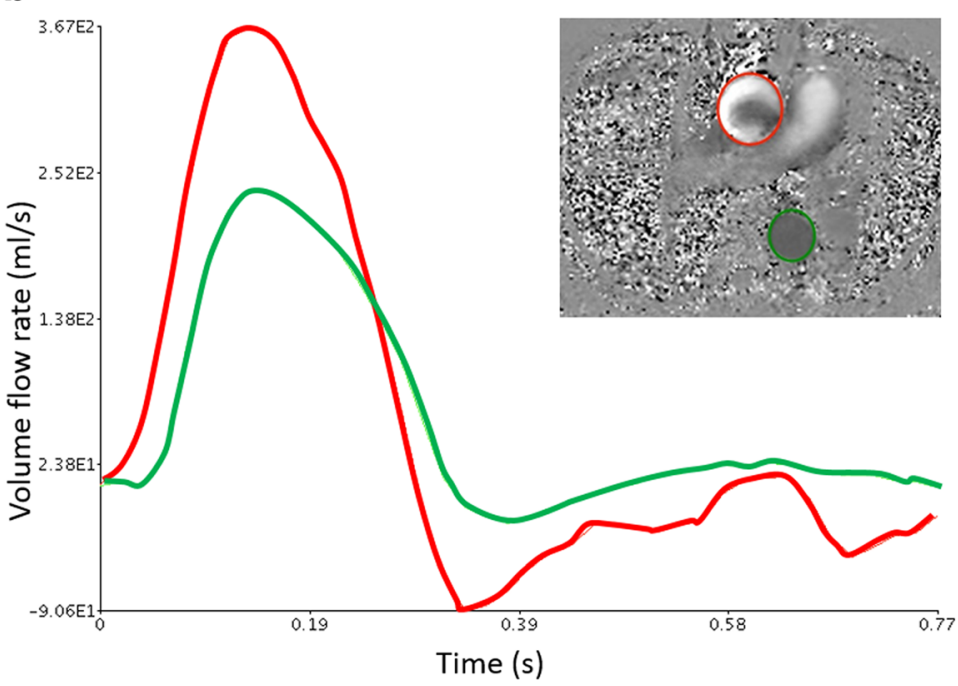

transit delay, which was estimated from the cross-correlation between the flow rate curves for the ascending (red) and descending (green) aorta (see text for details) 
Table 1 Demographic, echocardiographic, and MRI data for those with bicuspid and tricuspid aortic stenosis

\begin{tabular}{|c|c|c|c|c|c|c|}
\hline Variable & $\begin{array}{l}\text { Bicuspid } \\
(n=63)\end{array}$ & $\begin{array}{l}\text { Tricuspid } \\
(n=106)\end{array}$ & $\begin{array}{l}p \text { value } \\
\text { (bi vs tri) }\end{array}$ & $\begin{array}{l}\text { All AS } \\
(n=169)\end{array}$ & $\begin{array}{l}\text { Controls } \\
(n=23)\end{array}$ & $\begin{array}{l}p \text { value } \\
\text { (AS vs control) }\end{array}$ \\
\hline Age (years) & $64.6[51.1,69.7]$ & $71.4[65.8,77.3]$ & $<0.001 *$ & $69.3[61.5,75.6]$ & $66.0[61.0,74.8]$ & 0.935 \\
\hline Male (\%) & 73.0 & 77.4 & 0.524 & 75.7 & 69.6 & 0.521 \\
\hline $\operatorname{BSA}\left(\mathrm{m}^{2}\right)$ & $1.92 \pm 0.20$ & $1.97 \pm 0.20$ & 0.166 & $1.95 \pm 0.20$ & $1.93 \pm 0.18$ & 0.685 \\
\hline Hypertension (\%) & 44.4 & 60.4 & $0.044 *$ & 54.4 & 26.1 & $0.011 *$ \\
\hline Hyperlipidaemia (\%) & 47.6 & 58.5 & 0.390 & 54.4 & 21.7 & $<0.001 *$ \\
\hline Diabetes $(\%)$ & 7.9 & 17.9 & 0.072 & 14.2 & 8.7 & 0.469 \\
\hline Statin $(\%)$ & 49.2 & 67.9 & $0.016^{*}$ & 60.9 & 43.5 & 0.110 \\
\hline ACEI/ARB (\%) & 42.9 & 47.2 & 0.586 & 45.6 & 21.7 & $0.030 *$ \\
\hline B-blocker (\%) & 34.9 & 30.2 & 0.524 & 32.0 & 4.3 & $0.006 *$ \\
\hline HR (bpm) & $72.1 \pm 10.7$ & $69.3 \pm 11.6$ & 0.119 & $70.3 \pm 11.3$ & $72.6 \pm 8.2$ & 0.364 \\
\hline SBP (mmHg) & $142.7 \pm 21.3$ & $149.3 \pm 20.6$ & 0.050 & $146.8 \pm 21.1$ & $154.1 \pm 25.0$ & 0.132 \\
\hline DBP (mmHg) & $80.6 \pm 11.0$ & $74.9 \pm 9.9$ & $0.001 *$ & $77.0 \pm 10.6$ & $82.0 \pm 9.4$ & $0.032 *$ \\
\hline $\mathrm{PP}(\mathrm{mmHg})$ & $62.01 \pm 16.4$ & $74.41 \pm 19.9$ & $<0.001 *$ & $69.8 \pm 19.5$ & $72.02 \pm 24.0$ & 0.621 \\
\hline \multicolumn{7}{|l|}{ Echocardiographic data } \\
\hline $\mathrm{AV} \operatorname{Vmax}(\mathrm{m} / \mathrm{s})$ & $3.96 \pm 0.56$ & $3.79 \pm 0.55$ & 0.057 & $3.86 \pm 0.56$ & $1.36 \pm 0.27$ & $<0.001 *$ \\
\hline Mean gradient (mmHg) & $37.9 \pm 13.1$ & $33.7 \pm 11.6$ & $0.032 *$ & $35.3 \pm 12.3$ & $4.2 \pm 1.6$ & $<0.001 *$ \\
\hline $\operatorname{AVAI}\left(\mathrm{cm}^{2} / \mathrm{m}^{2}\right)$ & $0.58 \pm 0.16$ & $0.57 \pm 0.13$ & 0.773 & $0.57 \pm 0.14$ & $1.71 \pm 0.36$ & $<0.001 *$ \\
\hline $\mathrm{E} / \mathrm{A}$ & $0.92 \pm 0.32$ & $0.85 \pm 0.27$ & 0.204 & $0.88 \pm 0.29$ & $0.84 \pm 0.23$ & 0.523 \\
\hline Septal E/e' & $12.14 \pm 4.62$ & $12.44 \pm 5.02$ & 0.734 & $12.33 \pm 4.86$ & $10.67 \pm 3.34$ & 0.115 \\
\hline Lateral E/e' & $9.24 \pm 3.96$ & $10.21 \pm 3.63$ & 0.073 & $9.86 \pm 3.77$ & $8.07 \pm 2.97$ & $0.031 *$ \\
\hline \multicolumn{7}{|l|}{ MRI data } \\
\hline LVEDVI (ml/m²) & $88.3 \pm 18.4$ & $86.4 \pm 18.1$ & 0.505 & $87.1 \pm 18.2$ & $78.2 \pm 9.4$ & $0.001 *$ \\
\hline $\operatorname{LVEF}(\%)$ & $57.0 \pm 4.1$ & $56.7 \pm 5.1$ & 0.668 & $56.8 \pm 4.8$ & $58.9 \pm 3.7$ & 0.050 \\
\hline LVMI $\left(\mathrm{g} / \mathrm{m}^{2}\right)$ & $56.5[47.2,66.4]$ & $54.9[47.7,64.5]$ & 0.649 & $55.6[47.6,65.6]$ & $42.2[39.7,47.9]$ & $<0.001 *$ \\
\hline LV mass/volume & $0.66 \pm 0.12$ & $0.67 \pm 0.11$ & 0.631 & $0.66 \pm 0.11$ & $0.57 \pm 0.08$ & $<0.001 *$ \\
\hline LGE (g) & $2.67[0.77,4.96]$ & $2.20[0.83,6.22]$ & 0.904 & $2.44[0.81,6.08]$ & $0.77[0.40,2.22]$ & $0.001 *$ \\
\hline $\operatorname{ECV}(\%)$ & $24.48 \pm 2.46$ & $24.91 \pm 2.33$ & 0.350 & $24.8 \pm 2.4$ & $25.05 \pm 2.57$ & 0.590 \\
\hline Annulus (mm) & $25.53 \pm 2.69$ & $23.76 \pm 2.44$ & $<0.001 *$ & $24.42 \pm 2.67$ & $22.82 \pm 2.23$ & $0.006 *$ \\
\hline SoV (mm) & $34.82 \pm 4.00$ & $32.92 \pm 3.81$ & $0.002 *$ & $33.63 \pm 3.98$ & $33.50 \pm 3.78$ & 0.880 \\
\hline STJ (mm) & $29.52 \pm 3.88$ & $27.16 \pm 3.67$ & $<0.001 *$ & $28.04 \pm 3.91$ & $27.41 \pm 3.00$ & 0.456 \\
\hline Proximal AA (mm) & $36.40 \pm 5.22$ & $31.42 \pm 4.13$ & $<0.001^{*}$ & $33.27 \pm 5.15$ & $29.75 \pm 2.93$ & $<0.001 *$ \\
\hline
\end{tabular}

Data presented as mean, with standard deviation in parentheses or median with 25 th and 75 th centile in parenthesis

$S B P$ systolic blood pressure, $D B P$ diastolic blood pressure, $A V A I$ aortic valve area indexed to body surface area, $M R I$ magnetic resonance imaging, $L V E D V I$ left ventricular end-diastolic volume index, $L V M I$ left ventricular mass index, $L V E F$ left ventricular ejection fraction, $S o V$ sinus of valsalva, $S T J$ sinotubular junction, $A A$ ascending aorta

*Significant difference on unpaired $t$ test or Mann Whitney-U test, as appropriate

in AA distensibility, DA distensibility or PWV between bicuspid and tricuspid patients (Table 2). In addition, AA area did not correlate with AA distensibility (Fig. 3d).

\section{Comparison by bicuspid type-I, and type-II sub-groups}

There were no significant differences in age, gender, resting haemodynamics, LV function or AS severity between the two BAV sub-groups. There were significant differences in agecorrected AA area, AA distensibility and PWV between the three groups (tricuspid, bicuspid type-I, and type-II) (Table 3 and Fig. 4). Post-HOC analysis revealed a significantly higher AA area in both bicuspid sub-groups compared to tricuspid patients, and a significantly higher AA distensibility and lower PWV in the bicuspid type-II sub-group compared to bicuspid type-I patients. There were no significant differences in the DA area or distensibility between the sub-groups.

\section{Associations of AA distensibility and PWV}

The univariate associations of AA distensibility and PWV in the patient group, both before and after adjusting for age, are 
Table 2 Aortic area, distensibility, and pulse wave velocity in bicuspid and tricuspid groups

\begin{tabular}{lccccc}
\hline & Bicuspid $(n=63)$ & Tricuspid $(n=106)$ & $\begin{array}{l}p \text { value } \\
\text { (bi vs tri) }\end{array}$ & $\begin{array}{l}p \text { value after correcting } \\
\text { for age (bi vs tri) }\end{array}$ & Controls $(n=23)$ \\
\hline AAmax $\left(\mathrm{cm}^{2}\right)$ & $12.97[11.10,15.59]$ & $10.06[8.57,12.04]$ & $<0.001^{*}$ & $<0.001^{*}$ & $9.46[7.85,10.48]^{\dagger}$ \\
DAmax $\left(\mathrm{cm}^{2}\right)$ & $5.84[4.76,6.59]$ & $6.07[5.17,7.32]$ & $0.020^{*}$ & 0.902 & $5.18[4.68,5.92]^{\dagger}$ \\
AAmin $\left(\mathrm{cm}^{2}\right)$ & $11.57[10.08,14.11]$ & $9.00[7.56,10.66]$ & $<0.001^{*}$ & $<0.001^{*}$ & $8.98[7.24,9.49]^{\dagger}$ \\
DA min $\left(\mathrm{cm}^{2}\right)$ & $5.27[4.07,5.74]$ & $5.52[4.67,6.66]$ & $0.013^{*}$ & 0.980 & $4.54[4.27,5.53]^{\dagger}$ \\
AA distensibility $\left(10^{-3} \mathrm{mmHg}^{-1}\right)$ & $1.64[1.17,3.02]$ & $1.58[1.20,2.07]$ & 0.396 & 0.099 & $1.23[0.73,1.73]^{\dagger}$ \\
DA distensibility $\left(10^{-3} \mathrm{mmHg}^{-1}\right)$ & $2.45[1.43,2.89]$ & $1.55[1.10,2.18]$ & $<0.001^{*}$ & 0.498 & $1.40[1.05,1.95]$ \\
PWV $(\mathrm{m} / \mathrm{s})$ & $7.44[4.88,10.51]$ & $7.88[6.32,9.92]$ & 0.150 & 0.235 & $8.20[6.83,8.97]$ \\
\hline
\end{tabular}

Data presented as median [25th, 75th percentile]. Column-4 shows $p$ values using the Mann-Whitney $U$ test; column-5 shows $p$ values after correcting for age, using one-way ANCOVA test of log-transformed data $* p<0.05$

AAmax/min maximum/minimum ascending aortic area, DAmax/min maximum/ minimum descending aortic area, $P W V$ pulse wave velocity ${ }^{\dagger} p<0.05$ for comparison of controls with AS patients after correcting for age using one-way ANCOVA test of log-transformed data

summarised in supplemental Tables 1 and 2 . Age was the only independent factor associated with both parameters after entering the following variables into a stepwise multivariable model: age, sex, BMI, valve subtype, diabetes, hypertension (or PP instead for PWV), and eGFR.

\section{Discussion}

Our results show no statistically significant differences in aortic distensibility and PWV between bicuspid and tricuspid patients with AS, despite increased AA diameters in those with BAV. On further analysis, we found bicuspid type-II patients to have higher AA distensibility and lower aortic stiffness (PWV) than their type-I counterparts, despite a trend towards the highest AA area. The strengths of this study are the multicentre design, core lab blinded analysis, moderately large population, classification of valve morphology, and measurement of aortic stiffness with MRI, which allowed localised assessment in the area of interest.

\section{Aortic area}

We have confirmed findings from previous echocardiographic studies that patients with BAV have a greater degree of AA dilatation compared to tricuspid controls, either with or without valvular stenosis or regurgitation [21], and out of proportion to the degree of valvular dysfunction [2, 22]. Several studies have also looked at the relationship between the morphology of BAV and aortic dimensions, with mixed findings. Novaro et al found type-II BAV patients to have slightly higher mid-ascending aortic dimensions compared to type-I, though this did not reach statistical significance [23]. Type-II BAVs have also been associated with a larger aortic arch and ascending aorta [20]. However, Cecconi et al found no difference in aortic dimensions in 162 younger patients with type- 1 and type-II BAV, although the average age of that cohort was only 23.6 years and aortic dimensions did strongly correlate with age [24].

\section{Aortic stiffness}

Distensibility and PWV are markers of arterial wall stiffness that are inversely related according to the Bramwell and Hill equation [25] and reflect the elastic properties of the aorta. Aortic distensibility is principally governed by the composition of the aortic wall intra-cellular matrix and luminal mean arterial pressure. Previous MRI studies have also shown that distensibility decreases and PWV increases with age $[17,26$, 27], which is the likely reason for the stiffness parameters in our controls, who were older compared to the BAV group. Echocardiographic and MRI studies have found lower distensibility and higher aortic stiffness parameters in BAV patients without significant stenosis or regurgitation, compared to controls $[11,13]$. In one echocardiographic study of $32 \mathrm{BAV}$ patients with AS and 32 controls, aortic stiffness index was higher in the BAV group, but there was no significant difference in distensibility between the groups [12]. This suggests that the presence of stenosis may result in progressively reduced distensibility and increased PWV in both bicuspid and tricuspid patients, leading to no significant difference between the two groups. Support for this also comes from marked improvement in aortic stiffness in patients with severe AS 1 year after aortic valve replacement [28].

\section{Pathophysiology of aortic dilatation}

The exact mechanism leading to dilatation of the aorta in bicuspid patients is unclear. Histopathological studies have demonstrated changes in the AA walls of bicuspid patients including cystic medial necrosis [6]. High rates of apoptosis in the aortic media of bicuspid patients both with and without 

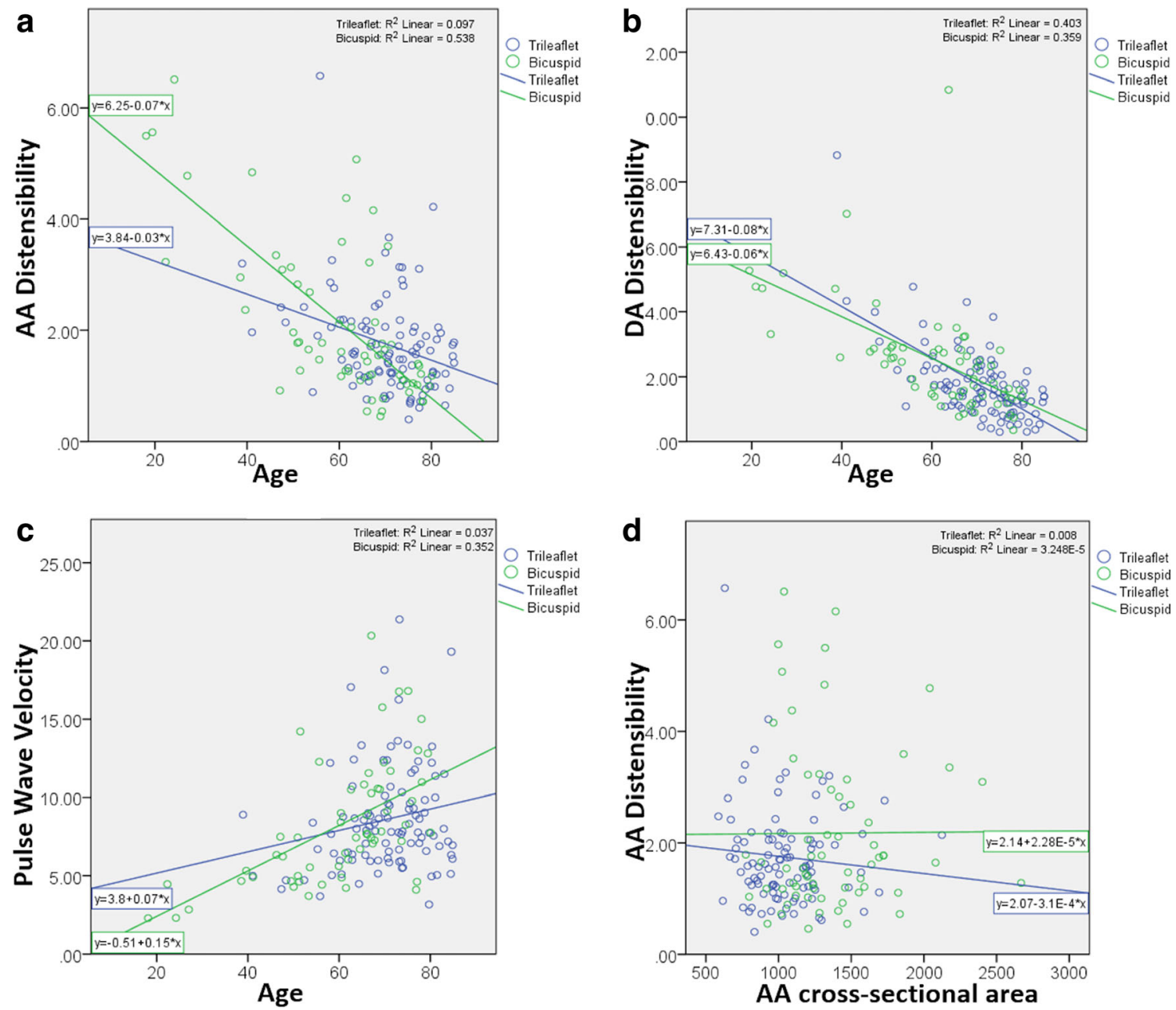

Fig. 3 Scatter plots showing the relationship of age with distensibility $(\mathbf{a}, \mathbf{b})$ and pulse wave velocity $(\mathbf{c})$ and of distensibility with cross-sectional area in the ascending aorta $(\mathbf{d})$. Units - distensibility, $10^{-3} \mathrm{mmHg}^{-1} ; \mathrm{PWV}, \mathrm{m} / \mathrm{s} ; \mathrm{AA}$ area, $\mathrm{mm}^{2}$; age years

Table 3 Aortic area, distensibility, and pulse wave velocity in tricuspid, bicuspid type-I, and type-II sub-group comparison using age as a covariate

$\begin{array}{lllll}\begin{array}{l}\text { Tricuspid } \\ (n=106)\end{array} & \begin{array}{l}\text { Bicuspid type-I } \\ (n=41)\end{array} & \begin{array}{l}\text { Bicuspid type-II } \\ (n=22)\end{array} & \begin{array}{l}p \text { value (AS } \\ \text { sub-groups only) }\end{array} & \begin{array}{l}\text { Controls } \\ (n=23)\end{array}\end{array}$

$p$ value (AS

sub-groups

and controls)

\begin{tabular}{lccccc}
\hline AA max $\left(\mathrm{cm}^{2}\right)$ & $10.06[8.57,12.04]^{\dagger *}$ & $12.82[10.62,14.95]$ & $13.28[11.97,16.20]$ & $<0.001^{*}$ & $9.46[7.85,10.48]^{\dagger *}<0.001^{*}$ \\
DA max $\left(\mathrm{cm}^{2}\right)$ & $6.07[5.17,7.32]$ & $5.89[4.87,6.60]$ & $5.32[4.05,6.54]$ & 0.953 & $5.18[4.68,5.92]$ \\
& & & & 0.102 \\
$\begin{array}{c}\text { AA distensibility } \\
\left(10^{-3} \mathrm{mmHg}^{-1}\right)\end{array}$ & $1.58[1.20,2.07]^{\dagger}$ & $1.47[1.02,1.79]^{*}$ & $2.60[1.27,3.51]$ & $0.010^{*}$ & $1.23[0.73,1.73]^{\circ *}<0.001^{*}$ \\
$\begin{array}{c}\text { DA distensibility } \\
\left(10^{-3} \mathrm{mmHg}^{-1}\right)\end{array}$ & $1.55[1.10,2.18]$ & $2.26[1.40,2.89]$ & $2.51[1.63,2.84]$ & 0.729 & $1.40[1.05,1.95]$ \\
PWV $(\mathrm{m} / \mathrm{s})$ & $7.88[6.32,9.92]^{\dagger}$ & $8.94[6.34,11.96]^{*}$ & $4.99[4.61,7.06]$ & $0.001^{*}$ & $8.20[6.83,8.97]$
\end{tabular}

Abbreviations same as for Table 2. Data presented as median [25th, 75th centile]. Age-corrected $p$ values obtained using ANCOVA test of logtransformed values with age as a covariate

$* p<0.05$

${ }^{\dagger}$ Significant difference compared to bicuspid type-I

* Significant difference compared to bicuspid type-II

oSignificant difference compared to tricuspid on post-HOC analysis using Bonferroni test 
a

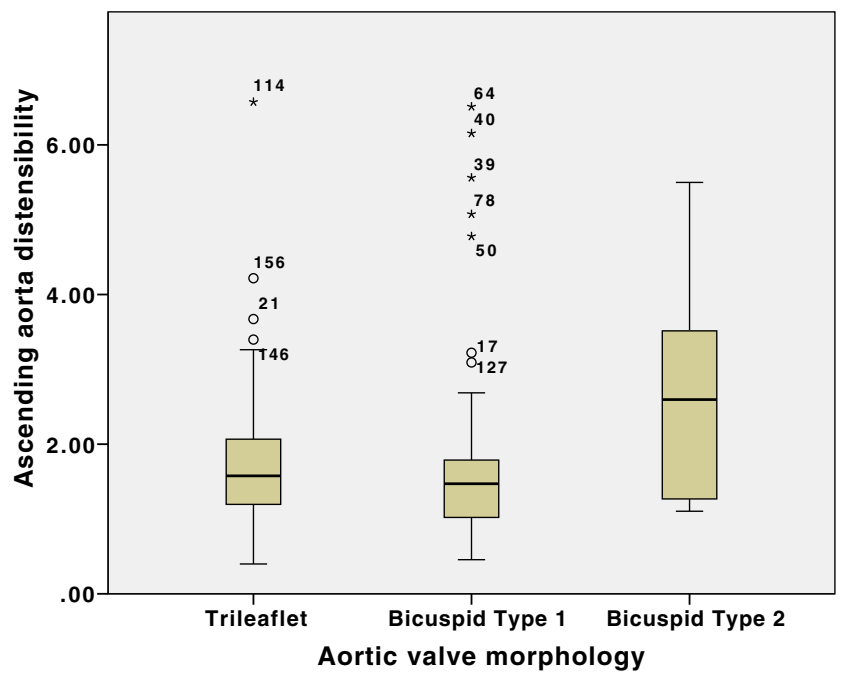

C

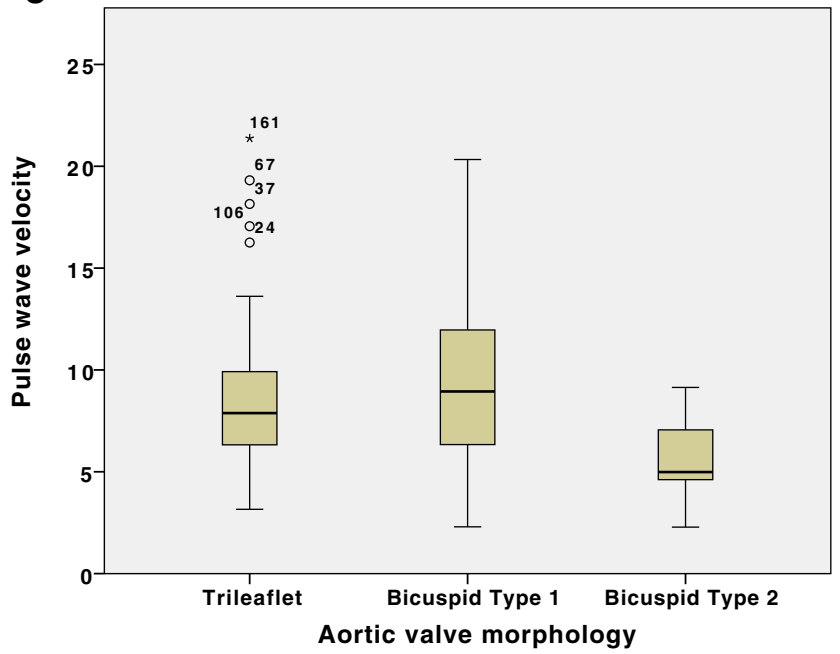

b

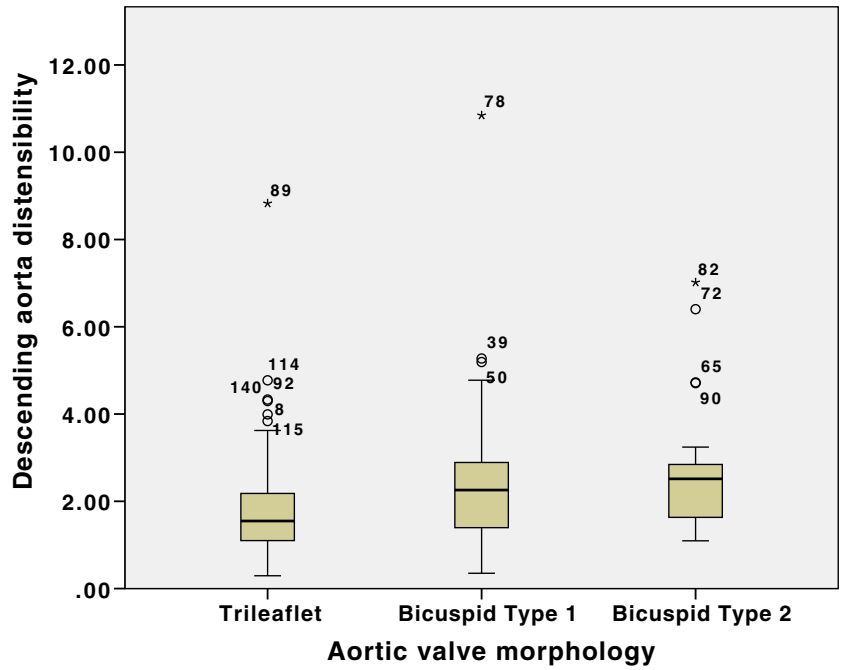

d

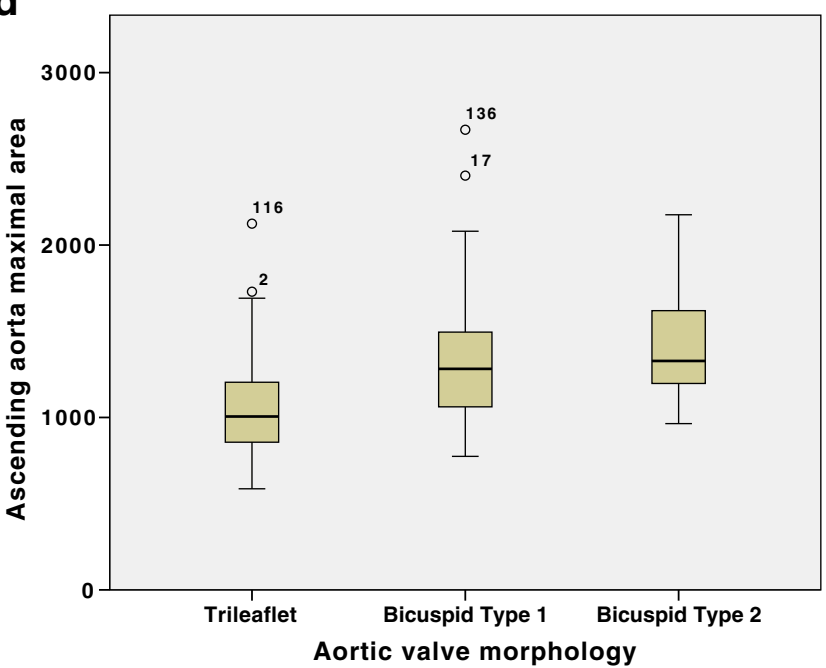

Fig. 4 Box plots showing the ascending aorta distensibility (a), descending aorta distensibility (b), pulse wave velocity (c), and maximum ascending aorta area (d) for patients with tricuspid, bicuspid type-I, and bicuspid type-II aortic valves. Units as in Fig. 3

dilatation have been shown, suggesting that apoptosis is a key mechanism for smooth muscle cell loss in the ascending aortas of bicuspid patients, and supporting the hypothesis of a developmental fault involving the valve and aortic wall [5]. However, a similarly high rate of apoptosis was also found in tricuspid patients with aortic dilatation, suggesting a role of extrinsic forces on the aortic wall, rather than an intrinsic developmental abnormality alone. Cystic medial necrosis can be found in hereditary connective tissue disorders such as Marfan syndrome, which has consistently been associated with increased aortic stiffness [29, 30], but a similar histological picture can also be caused by infection, atherosclerosis or severe shear stress [5].

We have shown a dissociation between aortic dilatation and stiffness in BAV disease, with type-II group having lower stiffness parameters, despite a trend towards a higher AA area, further confounding the theory of intrinsic aortic wall stiffness alone leading to aortic dilatation in bicuspid patients. In fact, there was no correlation between AA distensibility and AA dimensions (Fig. 3d). This may suggest a more central role of asymmetrical flow patterns and worse turbulence [31] that has been demonstrated in BAV compared to TAV [7, 8], which also correlated with the degree of proximal aortic dilatation [32]. Recently, time-resolved three-dimensional phasecontrast MRI, also called 4D flow, has demonstrated righthanded helical flow and right-anterior flow jets in type-I $\mathrm{BAV}$, and left-handed helical flow with left-posterior flow jets in type-II BAV [31].

In abdominal aortic aneurysms (AAA), Wilson et al demonstrated increasing aortic distensibility to be an independent predictor of rupture and suggested that this may be due to failure of aortic wall remodelling, which leads to further dilatation and risk of rupture [33]. This is supported by another study showing no correlation between AAA distensibility and 
size [34]. The authors speculated that the wall of the rapidly expanding AAA may lose its integrity leading to a paradoxically increased distensibility, which may also in part explain our findings in type-II BAV patients.

There appear to be two key players: age being a key determinant of aortic stiffness, with valve haemodynamics (morphology \pm presence of stenosis/regurgitation) being central to aortic dilatation. In a previously published set of young controls using the same methodology, the median AA distensibility was $6.36 \times$ $10^{-3} \mathrm{mmHg}^{-1}$ and PWV was $3.97 \mathrm{~m} / \mathrm{s}$ [35], demonstrating much lower stiffness than this group of patients or older controls. Cecconi found no difference in aortic size between young BAV type-I, and type-II patients with no stenosis/regurgitation [24], whereas type-II BAV patients with mixed valve disease (dysfunctional valves) had larger aortas but similar distensibility to type-I patients in Schaefer's study [36]. Finally, our older cohort of BAV and TAV with AS demonstrated no significant difference in aortic stiffness, despite larger aortas in BAV.

\section{Limitations}

The cross-sectional nature of the observations precludes any inference about the causality of the associated observations, and the results should be considered hypothesis generating. The bicuspid group were younger, and age is an important determinant of aortic stiffness. However, BAV presents at an earlier age, and we corrected for age in our analysis to try and overcome this limitation. We cannot exclude the possibility that the omission from the study of younger patients with BAV and complications, such as aortic dissection, potentially biased the results by excluding those at highest risk. Sub-group analysis of BAV was limited with relatively small sample size. Four-dimensional flow sequences were not acquired and therefore we could not correlate flow pattern with measures of stiffness. We also did not correct for longitudinal motion of the aortic root during systole, though the measurement was made more distally in the ascending aorta.

\section{Conclusions}

In patients with significant AS, BAV patients do not have increased aortic stiffness compared to those with TAV despite increased ascending aortic dimensions. The AA of patients with type-II BAV has the highest distensibility and lowest PWV despite the greatest dimensions. These results demonstrate a dissociation between aortic dilatation and stiffness and suggest that altered flow patterns may play a role which requires further assessment with longitudinal studies.
Acknowledgements We acknowledge all the research nurses and Fellows at each site for their contribution to recruitment for this study, as well as the University of Leicester Clinical Trials Unit for providing trial management support.

Funding The study was funded by a Post-Doctoral Fellowship supported by the National Institute for Health Research (NIHR-PDF 2011-04-51 Gerald P McCann). Support was also received from NIHR Leicester Cardiovascular Biomedical Research Unit, the NIHR Comprehensive Local Research Networks, and the Leeds and Leicester NIHR Clinical Research Facilities.

\section{Compliance with ethical standards}

The views expressed in this publication are those of the authors and not necessarily those of the NHS, the National Institute for Health Research or the Department of Health, UK.

Guarantor The scientific guarantor of this publication is Professor Gerry McCann.

Conflict of interest MAH is the owner of Xinapse Systems software which was used to quantify distensibility and PWV in this study. There are no other competing interests to declare.

Statistics and biometry No complex statistical methods were necessary for this paper.

Informed consent Written informed consent was obtained from all subjects before participation.

Ethical approval The study was approved by the United Kingdom National Research Ethics Service (11/EM/0410).

Study subjects or cohort overlaps Some study subjects have been previously reported in the main results of the PRIMID-AS study (as mentioned in reference 15 under the "Materials and methods" section of the manuscript). However, the aortic stiffness parameters are novel in this manuscript, as is the comparison of bicuspid and tricuspid sub-groups.

\section{Methodology}

- Prospective

- Multi-centre

- Observational study

Open Access This article is distributed under the terms of the Creative Commons Attribution 4.0 International License (http:// creativecommons.org/licenses/by/4.0/), which permits unrestricted use, distribution, and reproduction in any medium, provided you give appropriate credit to the original author(s) and the source, provide a link to the Creative Commons license, and indicate if changes were made.

\section{References}

1. Ward C (2000) Clinical significance of the bicuspid aortic valve. Heart 83:81-85

2. Ferencik M, Pape LA (2003) Changes in size of ascending aorta and aortic valve function with time in patients with congenitally bicuspid aortic valves. Am J Cardiol 92:43-46 
3. Michelena HI, Desjardins VA, Avierinos JF et al (2008) Natural history of asymptomatic patients with normally functioning or minimally dysfunctional bicuspid aortic valve in the community. Circulation 117:2776-2784

4. Guntheroth WG (2008) A critical review of the American College of Cardiology/American Heart Association practice guidelines on bicuspid aortic valve with dilated ascending aorta. Am J Cardiol 102:107-110

5. Bonderman D, Gharehbaghi-Schnell E, Wollenek G, Maurer G, Baumgartner H, Lang IM (1999) Mechanisms underlying aortic dilatation in congenital aortic valve malformation. Circulation 99: 2138-2143

6. Cecconi M, Nistri S, Quarti A et al (2006) Aortic dilatation in patients with bicuspid aortic valve. J Cardiovasc Med (Hagerstown) 7:11-20

7. Robicsek F, Sanger PW, Taylor FH, Magistro R, Foti E (1958) Pathogenesis and significance of post-stenotic dilatation in great vessels. Ann Surg 147:835-844

8. Robicsek F, Thubrikar MJ, Cook JW, Fowler B (2004) The congenitally bicuspid aortic valve: how does it function? Why does it fail? Ann Thorac Surg 77:177-185

9. Laurent S, Cockcroft J, Van Bortel L et al (2006) Expert consensus document on arterial stiffness: methodological issues and clinical applications. Eur Heart J 27:2588-2605

10. Grotenhuis HB, Westenberg JJ, Steendijk P et al (2009) Validation and reproducibility of aortic pulse wave velocity as assessed with velocity-encoded MRI. J Magn Reson Imaging 30:521-526

11. Nistri S, Sorbo MD, Basso C, Thiene G (2002) Bicuspid aortic valve: abnormal aortic elastic properties. J Heart Valve Dis 11: 369-373

12. Yap SC, Nemes A, Meijboom FJ et al (2008) Abnormal aortic elastic properties in adults with congenital valvular aortic stenosis. Int J Cardiol 128:336-341

13. Nistri S, Grande-Allen J, Noale M et al (2008) Aortic elasticity and size in bicuspid aortic valve syndrome. Eur Heart J 29:472-479

14. Grotenhuis HB, Ottenkamp J, Westenberg JJ, Bax JJ, Kroft LJ, de Roos A (2007) Reduced aortic elasticity and dilatation are associated with aortic regurgitation and left ventricular hypertrophy in nonstenotic bicuspid aortic valve patients. J Am Coll Cardiol 49: $1660-1665$

15. Singh A, Greenwood JP, Berry C et al (2017) Comparison of exercise testing and CMR measured myocardial perfusion reserve for predicting outcome in asymptomatic aortic stenosis: the PRognostic Importance of MIcrovascular Dysfunction in Aortic Stenosis (PRIMID AS) Study. Eur Heart J 38:1222-1229

16. Singh A, Ford I, Greenwood JP et al (2013) Rationale and design of the PRognostic Importance of MIcrovascular Dysfunction in asymptomatic patients with Aortic Stenosis (PRIMID-AS): a multicentre observational study with blinded investigations. BMJ Open 3:e004348

17. Voges I, Jerosch-Herold M, Hedderich J et al (2012) Normal values of aortic dimensions, distensibility, and pulse wave velocity in children and young adults: a cross-sectional study. J Cardiovasc Magn Reson 14:77

18. Singh A, Horsfield MA, Bekele S, Khan JN, Greiser A, McCann GP (2015) Myocardial T1 and extracellular volume fraction measurement in asymptomatic patients with aortic stenosis: reproducibility and comparison with age-matched controls. Eur Heart J Cardiovasc Imaging 16:763-770

19. Papavassiliu T, Kühl HP, Schröder M et al (2005) Effect of endocardial trabeculae on left ventricular measurements and measurement reproducibility at cardiovascular MR imaging. Radiology 236:57-64

20. Schaefer BM, Lewin MB, Stout KK et al (2008) The bicuspid aortic valve: an integrated phenotypic classification of leaflet morphology and aortic root shape. Heart 94:1634-1638

21. Nkomo VT, Enriquez-Sarano M, Ammash NM et al (2003) Bicuspid aortic valve associated with aortic dilatation: a community-based study. Arterioscler Thromb Vasc Biol 23:351356

22. Keane MG, Wiegers SE, Plappert T, Pochettino A, Bavaria JE, Sutton MG (2000) Bicuspid aortic valves are associated with aortic dilatation out of proportion to coexistent valvular lesions. Circulation 102:III35-III39

23. Novaro GM, Tiong IY, Pearce GL, Grimm RA, Smedira N, Griffin BP (2003) Features and predictors of ascending aortic dilatation in association with a congenital bicuspid aortic valve. Am J Cardiol 92:99-101

24. Cecconi M, Manfrin M, Moraca A et al (2005) Aortic dimensions in patients with bicuspid aortic valve without significant valve dysfunction. Am J Cardiol 95:292-294

25. Skaluba SJ, Litwin SE (2004) Mechanisms of exercise intolerance: insights from tissue Doppler imaging. Circulation 109:972-977

26. Ibrahim el-SH, Johnson KR, Miller AB, Shaffer JM, White RD (2010) Measuring aortic pulse wave velocity using high-field cardiovascular magnetic resonance: comparison of techniques. J Cardiovasc Magn Reson 12:26

27. Mohiaddin RH, Firmin DN, Longmore DB (1993) Age-related changes of human aortic flow wave velocity measured noninvasively by magnetic resonance imaging. J Appl Physiol (1985) 74: 492-497

28. Nemes A, Galema TW, Geleijnse ML et al (2007) Aortic valve replacement for aortic stenosis is associated with improved aortic distensibility at long-term follow-up. Am Heart J 153:147-151

29. Dalsgaard M, Kjaergaard J, Pecini R et al (2010) Predictors of exercise capacity and symptoms in severe aortic stenosis. Eur J Echocardiogr 11:482-487

30. Dalsgaard M, Kjaergaard J, Pecini R et al (2009) Left ventricular filling pressure estimation at rest and during exercise in patients with severe aortic valve stenosis: comparison of echocardiographic and invasive measurements. J Am Soc Echocardiogr 22:343-349

31. Hope MD, Hope TA, Meadows AK et al (2010) Bicuspid aortic valve: four-dimensional MR evaluation of ascending aortic systolic flow patterns. Radiology 255:53-61

32. den Reijer PM, Sallee D 3rd, van der Velden P et al (2010) Hemodynamic predictors of aortic dilatation in bicuspid aortic valve by velocity-encoded cardiovascular magnetic resonance. $\mathrm{J}$ Cardiovasc Magn Reson 12:4

33. Wilson KA, Lee AJ, Lee AJ et al (2003) The relationship between aortic wall distensibility and rupture of infrarenal abdominal aortic aneurysm. J Vasc Surg 37:112-117

34. Molacek J, Baxa J, Houdek K, Treska V, Ferda J (2011) Assessment of abdominal aortic aneurysm wall distensibility with electrocardiography-gated computed tomography. Ann Vasc Surg 25:1036-1042

35. Khan JN, Wilmot EG, Leggate M et al (2014) Subclinical diastolic dysfunction in young adults with Type 2 diabetes mellitus: a multiparametric contrast-enhanced cardiovascular magnetic resonance pilot study assessing potential mechanisms. Eur Heart J Cardiovasc Imaging 15:1263-1269

36. Schaefer BM, Lewin MB, Stout KK, Byers PH, Otto CM (2007) Usefulness of bicuspid aortic valve phenotype to predict elastic properties of the ascending aorta. Am J Cardiol 99:686-690 